\title{
Neoadjuvant Dose-Dense Gemcitabine plus Docetaxel and Vinorelbine plus Epirubicin for Operable Breast Cancer Improved Prognosis in Triple-Negative Tumors
}

Jacques Medioni, ${ }^{1,2}$ Cyrille Huchon, ${ }^{2,3}$ Marie-Aude Le Frere-Belda, ${ }^{4}$ Henri Hofmann, ${ }^{3,5}$ Anne-Sophie Bats, ${ }^{2,3}$ Denise Eme, Jean-Marie Andrieu, ${ }^{1,2}$ Stéphane Oudard, ${ }^{1,2,6}$ Fabrice Lecuru ${ }^{2,3}$ and Eric Levy ${ }^{1}$

1 Department of Medical Oncology, Georges Pompidou European Hospital, Paris, France

2 Paris Descartes University, Paris, France

3 Department of Gynecological and Oncological Surgery, Georges Pompidou European Hospital, Paris, France

4 Pathology Department, Georges Pompidou European Hospital, Paris, France

5 Surgery Service, Clinique Saint Jean de Dieu, Paris, France

6 EA 4054 University Paris Descartes, ENVA, Georges Pompidou European Hospital, Public Assistance Hospital of Paris, Paris, France

Background: Neoadjuvant anti-tumor activity of an alternating taxane- and anthracycline-based dose-dense regimen in patients with operable, noninflammatory large breast cancer was investigated.

Objective: The objective is to study the rate of pathological complete response in patients with breast cancer receiving dose-dense chemotherapy sequentially with gemcitabine plus docetaxel and vinorelbine plus epirubicin. Methods: Women $(n=74)$ with clinical stage II or III breast cancer were enrolled in this open-label, multicenter study to receive six 2-weekly courses of gemcitabine $1000 \mathrm{mg} / \mathrm{m}^{2}$ plus docetaxel $75 \mathrm{mg} / \mathrm{m}^{2}$ on days 1 and 15 , and vinorelbine $25 \mathrm{mg} / \mathrm{m}^{2}$ plus epirubicin $100 \mathrm{mg} / \mathrm{m}^{2}$ on days 29 and 43 . Patients with an objective response on day 56 then received another cycle of gemcitabine/docetaxel on day 57 and of vinorelbine/epirubicin on day 71. Conservative surgery was scheduled for all patients.

Results: Of the patients enrolled, 30\% had triple-negative breast cancer (TNBC). The pathologic complete response (pCR) rate was $22 \%$ overall, but was higher in TNBC than patients without TNBC (40.9\% vs $14.0 \%$; $\mathrm{p}=0.028$ ). Among patients with a $\mathrm{pCR}$, patients with TNBC had similar recurrence-free survival (RFS) and overall survival (OS) to patients without TNBC. Among those without a pCR, RFS rates for patients with TNBC were significantly lower than for patients without TNBC $(p=0.04)$. The most common severe hematologic toxicity was neutropenia. 
Conclusions: Administering four drugs in a dose-dense alternating sequence gave a high pCR in patients with operable, invasive breast cancer. Patients with TNBC with a pCR had similar OS to patients without TNBC, whereas patients with $\mathrm{TNBC}$ without a $\mathrm{pCR}$ had poorer survival rate than their nonTNBC counterparts.

\section{Introduction}

Neoadjuvant chemotherapy reduces tumor burden, thus allowing the possibility of breastconserving surgery. ${ }^{[1,2]}$ It is believed to treat clinically undetectable micrometastases ${ }^{[3]}$ and allows for monitoring of tumor response to therapy. ${ }^{[4]}$ Neoadjuvant therapy has recently become more commonly used in patients with larger tumors and/or lymph node involvement at diagnosis. Although no single chemotherapy regimen is specifically recommended over other regimens, regimens tend to be anthracycline-based with the addition of a taxane for high-risk, node-positive disease.

There is a clear association between breast cancer subtype and patient outcomes. Patients with 'triple-negative' breast cancer (TNBC), where tumors are estrogen receptor (ER)-, progesterone receptor (PR)-, and human epidermal growth factor receptor 2 (HER2)-negative tend to present with large, high-grade tumors. ${ }^{[5-8]} \mathrm{TNBC}$ is associated with aggressive clinical features, ${ }^{[9,10]}$ poor prognosis, ${ }^{[7,8,11]}$ and a high rate of local relapse. ${ }^{[12]}$ There is some evidence that TNBC is probably one of the more chemosensitive subtypes, ${ }^{[13,14]}$ but the most effective agents are currently unknown. ${ }^{[15]}$ The genetic instability of these TNBCs is expected to lead to an increased potential for resistance to treatments, which may eventually be reduced by the use of combination and sequential regimens. ${ }^{[15]}$ Dose-dense chemotherapy (2-weekly intervals between cycles) may improve long-term outcomes relative to standard 3-weekly schedules. ${ }^{[16]}$ Sequential chemotherapy allows dose intensity to be maintained in order to preserve efficacy and reduce toxicity, ${ }^{[16]}$ and the sequential administration of taxanes and anthracyclines has demonstrated high anti-tumor activity in the neoadjuvant setting. ${ }^{[17]}$
A pathologic complete response ( $\mathrm{pCR}$ ) rate of $26 \%$ was achieved in a small phase I/II study of docetaxel in combination with gemcitabine and epirubicin given as intensive (six cycles) neoadjuvant therapy in patients with early breast cancer, suggesting that this combination is highly active. ${ }^{[18]}$ Therefore, the anti-tumor activity of gemcitabine plus docetaxel and of vinorelbine plus epirubicin given as neoadjuvant therapy to patients with large, operable, invasive breast tumors was investigated. To achieve appropriate dose intensity of each agent whilst limiting cumulative toxicity, an alternating sequence of these two combination regimens was designed, which were given every 2 weeks.

First, a pilot safety study of the regimen in 13 patients with breast cancer was conducted, which demonstrated promising efficacy (own unpublished data). Given these encouraging results, the current study was conducted. We propose this regimen to be referred to as GTEN (Gemzar ${ }^{\circledR}$ Taxotere ${ }^{\circledR}$ Epirubicin Navelbine ${ }^{\circledR}$ ).

\section{Materials and Methods}

This was an open-label, prospective, phase II trial conducted in two centers in Paris, France. All patients provided written informed consent and the study was conducted with local Ethics Committee approval and in accordance with Good Clinical Practice and the Declaration of Helsinki.

\section{Patients}

Women aged 18-75 years with chemotherapynaïve, histologically-proven breast adenocarcinoma with a clinically-determined tumor size of $\geq 3 \mathrm{~cm}$ or local lymph node involvement, assessed clinically, and adequate blood cell counts, liver function, 
renal function and cardiac function were enrolled. Patients were excluded if they had inflammatory breast cancer, known overexpression (an immunohistochemical [IHC] score of $\geq 2+$ ) of HER2 or metastatic disease.

\section{Study Protocol}

Patients received between four and six 2-week cycles of chemotherapy, starting with two cycles of docetaxel $75 \mathrm{mg} / \mathrm{m}^{2}$ plus gemcitabine $1000 \mathrm{mg} / \mathrm{m}^{2}$ (cycle 1 on day 1 and cycle 2 on day 15) followed by two cycles of vinorelbine $25 \mathrm{mg} / \mathrm{m}^{2}$ plus epirubicin $100 \mathrm{mg} / \mathrm{m}^{2}$ (cycle 3 on day 29 and cycle 4 on day 43). Thereafter, patients without an objective response (determined clinically or radiologically), underwent surgery with axillary lymph node dissection; those with an objective response received one cycle of docetaxel plus gemcitabine at the same dose (cycle 5 on day 57) followed by one cycle of vinorelbine plus epirubicin at the same dose (cycle 6 on day 71). For patients who had received six cycles, at the final assessment on day 85 , patients without an objective response were treated off-protocol whilst those with an objective response underwent surgery with axillary lymph node dissection. Thus, all patients in the study were scheduled to undergo conservative surgery. Figure 1 summarizes the protocol description.

Treatment was delayed by 1 week for hematologic toxicity, defined as an absolute neutrophil count of $<1.0 \times 10^{9} / \mathrm{L}$ and/or platelet count of $<100 \times 10^{9} / \mathrm{L}$ in the 24 hours prior to treatment.

All patients received filgrastim $5 \mu \mathrm{g} / \mathrm{kg}$ subcutaneously from the 5th to 8th day after each chemotherapy infusion.

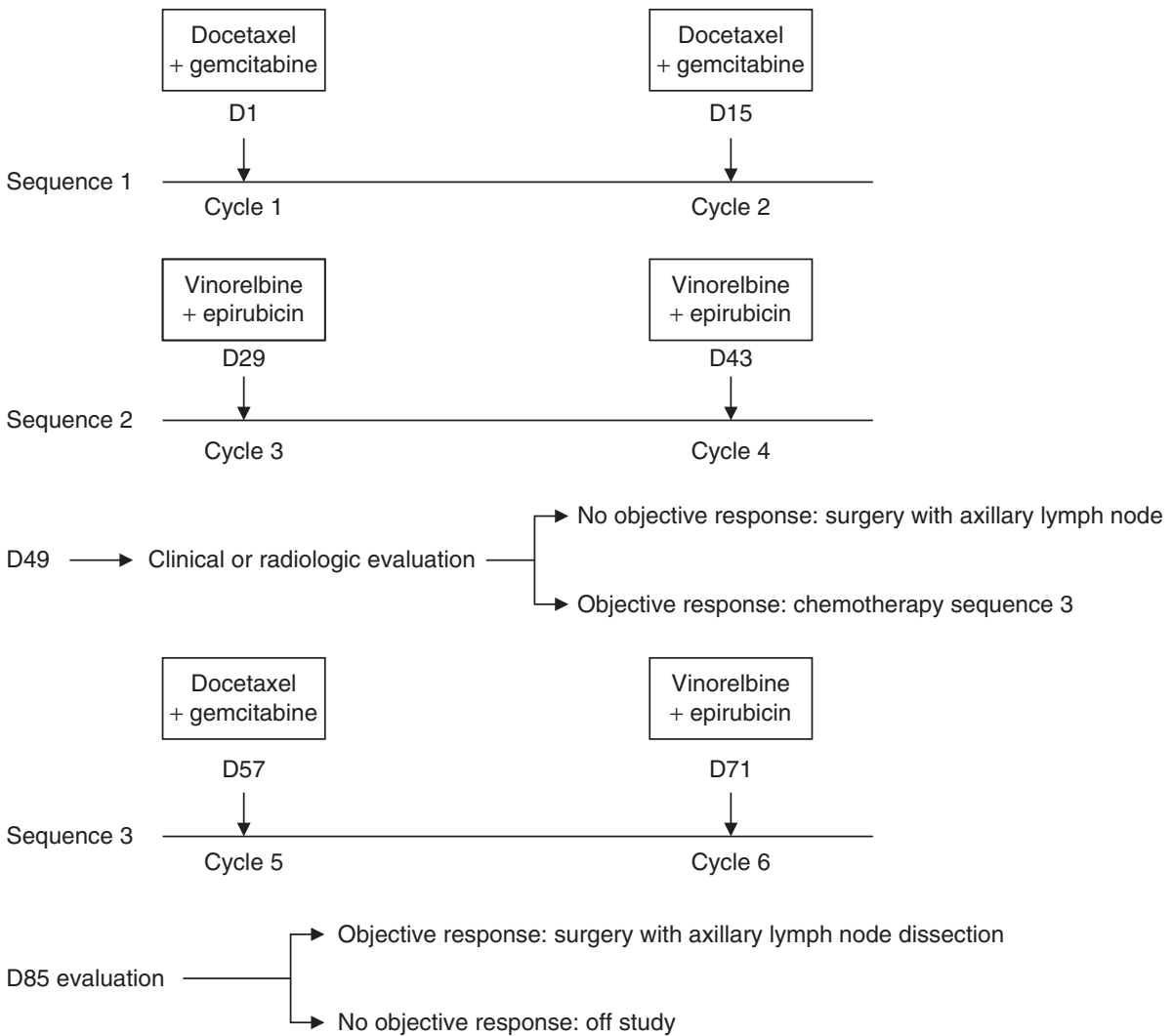

Fig. 1. The timing of chemotherapy administration and evaluation of tumor response. $\mathbf{D}=$ day. 


\section{Planned Interim Analyses}

Treatment response was assessed at two interim analyses (based on the Simon two-stage minimax design ${ }^{[19]}$ ) as follows: if more than three complete pathologic responses were observed in the first 21 evaluable patients at the first analysis, then a further 28 patients were enrolled. If the cumulative total number of pathologic responses was $\geq 11$ at the second analysis of evaluable patients (of the 49 enrolled), the treatment was considered effective and enrollment could continue. If at either analysis these criteria were not met, the study would be discontinued.

\section{Clinical Response Criteria}

The pCR was determined by microscopic examination of the excised tissues from the breast and regional lymph nodes, and was defined as no residual disease in either tissue. A lymph node response was defined as an absence of axillary lymph node involvement as determined by microscopic examination of excised tissue after treatment $(\mathrm{pN}-)$ in patients who had lymph node involvement at baseline $(\mathrm{N}+)$. The objective response by clinical or radiological criteria was determined according to Response Evaluation Criteria in Solid Tumors (RECIST 1.0). ${ }^{[20]}$

\section{Efficacy Endpoints and Assessments}

The primary efficacy endpoint was the proportion of patients with a pCR. Secondary efficacy endpoints included overall survival (OS) [defined as the time from tumor diagnosis to death], recurrence-free survival (RFS) [defined as patients alive and without recurrent disease as from the first day of tumor diagnosis to the time of first local or metastatic recurrence or tumor-related death], and the rate of metastatic and local recurrence. Exploratory analyses included the proportion of patients with residual disease, an analysis of lymph node response by baseline lymph node involvement, and IHC analyses of tissue from the initial biopsy for ER, PR, and HER2 status. ${ }^{[21]}$ ER (clone 6F11, diluted $1: 30$ ) and PR (clone PGR-312, diluted $1: 100)$ monoclonal antibodies were purchased from Novocastra (Newcastle upon
Tyne, UK), and HER2 (clone A0485, diluted $1: 600)$ from Dako (Glostrup, Denmark). An exploratory analysis was also conducted for treatment response and survival parameters in TNBC and non-TNBC.

Clinical tumor evaluation, breast ultrasound, and mammography were performed after the fourth and sixth cycles of chemotherapy.

\section{Safety and Laboratory Assessments}

Patients had a complete laboratory and vital sign (temperature, pulse, blood pressure) assessment 1 week prior to study inclusion and also during the study. Tests performed included a weekly complete blood count, vital signs measurement on treatment administration days, and blood biochemistry before each cycle, and measurement of cardiac parameters by ultrasound or scintigraphy after two cycles and at the end of every cycle thereafter. Adverse events and toxicities were assessed at every cycle according to National Cancer Institute Common Toxicity Criteria version 2. ${ }^{[22]}$

\section{Statistical Methods}

To achieve a statistical power of $80 \%$, assuming a minimum acceptable pCR rate of $15 \%$ and that $85 \%$ of patients would be evaluable, enrollment of 57 patients was required (in two stages, see the 'Planned Interim Analyses' section). The intent-to-treat (ITT) and safety populations included patients who received at least one cycle of study drug, and the per-protocol population included those evaluable for response and who received the scheduled study medication (at least three cycles of study drug).

Categorical variables were compared using the Chi-squared test or the Fisher exact test and continuous variables were compared using ANOVA between TNBC and non-TNBC.

We compared Kaplan-Meier curves for OS and RFS with the log-rank test for several variables (e.g. age, tumor size, positivity of lymph node, clinical stage, histologic grade, positivity of hormone receptors, TNBC subtypes, pCR). Unadjusted hazard ratios (HR) were calculated for each studied variable. All p-values were two-sided. 
Analyses were undertaken with Stata 9.2 (Stata corporation).

\section{Results}

Seventy-four patients were enrolled between December 2002 and December 2006, and all were included in the ITT and safety populations. One patient received chemotherapy but refused surgery, thus there were 73 patients in the per-protocol population. Patient baseline demographic and disease characteristics are given in table I. Most patients had invasive ductal tumors (89\%), tumornode-metastasis (TNM) clinical stage II (81\%), and Elston and Ellis histologic grade II (44\%) or III disease (23\%). Contrary to enrollment criteria, a small proportion of patients with HER2positive (HER2+) disease were enrolled (two patients expressed ++ HER2). Twenty-two patients had TNBC and 51 had non-TNBC (data missing

Table I. Patient (pt) clinical characteristics

\begin{tabular}{|c|c|c|c|c|}
\hline Characteristic & Total & $\mathrm{TNBC}^{\mathrm{a}}$ & Non-TNBC & $p-$ Value $^{b}$ \\
\hline No. of pts enrolled & 74 & 22 & 51 & \\
\hline \multicolumn{5}{|l|}{ Age $[\mathrm{n} / \mathrm{N}(\%)]$} \\
\hline$<50 y$ & $38 / 74(51.4)$ & $11 / 22(50.0)$ & $27 / 51(52.9)$ & \multirow[t]{2}{*}{0.82} \\
\hline$\geq 50 y$ & $36 / 74(48.6)$ & $11 / 22(50.0)$ & $24 / 51(47.1)$ & \\
\hline $\begin{array}{l}\text { Mean tumor size }[\mathrm{cm} \pm S D] \\
\text { (no. of pts with data available) }\end{array}$ & $\begin{array}{l}4.3 \pm 1.9 \\
(n=58)\end{array}$ & $\begin{array}{l}4.7 \pm 2.2 \\
(n=18)\end{array}$ & $\begin{array}{l}4.0 \pm 1.7 \\
(n=39)\end{array}$ & 0.24 \\
\hline $\mathrm{pN}+$ on clinical examination $[\mathrm{n} / \mathrm{N}(\%)]$ & $28 / 70(40.0)$ & $10 / 20(50.0)$ & $19 / 50(65.5)$ & 0.36 \\
\hline \multicolumn{5}{|l|}{ Clinical stage $^{\mathrm{c}}[\mathrm{n} / \mathrm{N}(\%)]$} \\
\hline I & $1 / 71(1.4)$ & $0 / 20(0)$ & $1 / 50(2.0)$ & \multirow[t]{3}{*}{1.00} \\
\hline II & $58 / 71(81.7)$ & $17 / 20(85.0)$ & $40 / 50(80.0)$ & \\
\hline III & $12 / 71(16.9)$ & $3 / 20(15.0)$ & $9 / 50(18.0)$ & \\
\hline \multicolumn{5}{|l|}{ Histologic tumor type [n/N (\%)] } \\
\hline invasive ductal & $66 / 74(89.2)$ & $21 / 22(95.4)$ & $45 / 51(88.2)$ & \multirow[t]{2}{*}{0.67} \\
\hline Other & $8 / 74(10.8)$ & $1 / 22(4.6)$ & $6 / 51(11.8)$ & \\
\hline \multicolumn{5}{|l|}{ Histologic grade ${ }^{d}[\mathrm{n} / \mathrm{N}(\%)]$} \\
\hline 1 & $8 / 74(10.8)$ & $2 / 22(9.0)$ & $6 / 51(11.7)$ & \multirow[t]{4}{*}{0.001} \\
\hline 2 & $33 / 74(44.6)$ & $3 / 22(13.6)$ & $30 / 51(58.8)$ & \\
\hline 3 & $17 / 74(23.0)$ & $10 / 22(45.4)$ & $7 / 51(13.7)$ & \\
\hline not specified & $16 / 74(21.6)$ & $7 / 22(32.6)$ & $8 / 51(15.7)$ & \\
\hline \multicolumn{5}{|l|}{ Estrogen receptor status [n/N (\%)] } \\
\hline Positive & $43 / 74(58.1)$ & $0 / 22(0)$ & $43 / 51(84.3)$ & \multirow[t]{2}{*}{$<0.001$} \\
\hline Negative & $31 / 74(41.9)$ & $22 / 22(100)$ & $8 / 51(26.7)$ & \\
\hline \multicolumn{5}{|l|}{ Progesterone receptor status [n/N (\%)] } \\
\hline Positive & $28 / 74(37.8)$ & $0 / 22(0)$ & $28 / 51(54.9)$ & \multirow[t]{2}{*}{$<0.001$} \\
\hline Negative & $46 / 74(62.2)$ & $22 / 22(100)$ & $23 / 51(45.1)$ & \\
\hline \multicolumn{5}{|l|}{ HER2 expression [n/N (\%)] } \\
\hline $2+/ 3+$ & $9 / 74(12.2)$ & $0 / 22(0)$ & $9 / 51(17.6)$ & \multirow[t]{3}{*}{0.05} \\
\hline $0 / 1+$ & $61 / 74(82.4)$ & $20 / 22(90.9)$ & $40 / 51(78.4)$ & \\
\hline Unknown & $4 / 74(5.4)$ & $2 / 22(9.1)$ & $2 / 51(3.9)$ & \\
\hline \multicolumn{5}{|c|}{ a For one patient, data are missing for the TNBC/non-TNBC status. } \\
\hline \multicolumn{5}{|c|}{ b $p$-Value of test comparing distribution of TNBC/non-TNBC for the various characteristics. } \\
\hline \multirow{2}{*}{\multicolumn{5}{|c|}{$\begin{array}{l}\text { c Clinical staging using American Joint Committee on Cancer tumor- } \\
\text { d Histologic grade using Elston and Ellis histologic classification. }{ }^{[23]}\end{array}$}} \\
\hline & & & & \\
\hline \multicolumn{5}{|c|}{ HER2 = human epidermal growth factor receptor 2; TNBC = triple-negative breast cancer. } \\
\hline
\end{tabular}


Table II. Treatment response and patient outcomes [n/N (\%)]

\begin{tabular}{|c|c|c|c|}
\hline Variable & Total & TNBC $^{a}$ & Non-TNBC \\
\hline \multicolumn{4}{|l|}{ Primary endpoint } \\
\hline $\mathrm{pCR}^{\mathrm{b}}$ & 16/73 (21.9) & $9 / 22(40.9)^{*}$ & $7 / 50(14.0)$ \\
\hline \multicolumn{4}{|l|}{ Exploratory endpoints } \\
\hline Residual disease & $57 / 73(78.1)$ & $12 / 22(54.6)^{*}$ & $43 / 50(86.0)$ \\
\hline Lymph node response $^{c}$ & $10 / 28(35.7)$ & $2 / 10(20.0)^{* * *}$ & $8 / 18(44.4)$ \\
\hline \multicolumn{4}{|l|}{ Secondary endpoints } \\
\hline Local recurrence & $1 / 74(1.4)$ & $0 / 22(0)$ & $1 / 51(2.0)$ \\
\hline Distant metastasis & $14 / 74(18.9)$ & 6/22 (27.3) & $7 / 51(13.7)$ \\
\hline Overall survival & $68 / 74(91.9)$ & 19/22 (86.4) & $48 / 51(94.1)$ \\
\hline Recurrence-free survival & $59 / 74(79.7)$ & 16/22 (72.7) & $43 / 51(84.3)$ \\
\hline \multicolumn{4}{|c|}{ a For one patient, data are missing for the TNBC/non-TNBC status. } \\
\hline \multicolumn{4}{|c|}{ b One patient did not undergo surgery. } \\
\hline \multicolumn{4}{|c|}{$\begin{array}{l}\text { c Patients with node-positive disease (N1, N2, or N3) by clinical } \\
\text { examination at baseline who were classified pN0 by pathologic } \\
\text { examination after treatment and had a lymph node response. }\end{array}$} \\
\hline \multicolumn{4}{|c|}{$\begin{array}{l}\text { PCR }=\text { pathologic complete response; TNBC }=\text { triple-negative breast } \\
\text { cancer; }{ }^{*} p=0.028 \text { vs non-TNBC (Fisher's Exact test), }{ }^{* *} p=0.25 \text { vs } \\
\text { non-TNBC (Fisher's Exact test). }\end{array}$} \\
\hline
\end{tabular}

for one patient). Histologic grade was significantly higher in TNBC than patients without $\operatorname{TNBC}(\mathrm{p}=0.001)$.

\section{Treatment}

Three patients had at least three cycles of chemotherapy. Five patients received the first four cycles of chemotherapy but proceeded to surgery thereafter because they did not have an objective response at the first assessment. Sixty-five patients received more than four cycles, of whom 35 underwent mastectomy and 30 breast-conserving surgery; all patients had lymph node dissection. Eight patients discontinued chemotherapy because of adverse events and therefore did not receive all six chemotherapy cycles. At the final assessment (day 85), only one patient was without an objective response and was treated off-protocol by surgery.

Mean cumulative doses were docetaxel $215 \mathrm{mg} / \mathrm{m}^{2}$ (95\% of planned dose), gemcitabine $2878 \mathrm{mg} / \mathrm{m}^{2}$ (95\% of planned dose), vinorelbine $68 \mathrm{mg} / \mathrm{m}^{2}$ (90\% of planned dose), and epirubicin $275 \mathrm{mg} / \mathrm{m}^{2}$ (91\% of planned dose).

All patients $(n=32)$ who underwent tumorectomy received radiotherapy, as well as those who underwent mastectomy with $\mathrm{T} 3$ or greater tumor size or with three or more lymph node involvement. No further chemotherapy was prescribed. All patients with positive ER and/or PR received hormonal therapy. Two patients with HER over-expression received adjuvant trastuzumab.

\section{Treatment Response}

A pCR was observed in $21.9 \%$ of patients (table II). Of the 28 of 70 patients who had positive lymph nodes on clinical examination, over one-third of patients had a lymph node response. Of note, pCR occurred in significantly more TNBC than patients without TNBC $(40.9 \%$ vs $14.0 \% ; \mathrm{p}=0.028)$. The corresponding proportion of patients with residual disease was therefore significantly lower in the TNBC than non-TNBC group $(\mathrm{p}=0.028)$. More patients with a lymph node response tended to have non-TNBC than TNBC disease (44.4\% vs $20.0 \%$ ), although this difference was not significant.

Most of the patients with no lymph node involvement prior to neoadjuvant therapy remained node-negative at surgery (35 of 42 patients). Seven patients with clinically lymph node negative at baseline, had lymph node positive, diagnosed pathologically, after surgery. Of the 28 patients with node-positive disease prior to treatment, ten had no evidence of disease at surgery (table III). Moreover, the proportion of patients who experienced a local recurrence was very low (1.4\%), and distant metastases were reported in $19 \%$ of patients after neoadjuvant therapy (table II). The difference between patients with TNBC and non-TNBC for these two endpoints was not significant.

\section{Survival Parameters}

After a median follow-up of 3.18 years, OS and RFS rates were $91.9 \%$ and $79.7 \%$, respectively

Table III. Lymph node response to treatment by baseline lymph node status in evaluable patients (pts) $[n=70]$

\begin{tabular}{llll}
\hline Pathologic lymph node & \multicolumn{3}{l}{ Clinical lymph node status at baseline $(\mathrm{n})$} \\
\cline { 2 - 4 }$(\mathrm{pN})$ status at surgery & $\mathrm{N}-$ & $\mathrm{N}+$ & total no. of pts \\
\hline $\mathrm{pN}-$ & 35 & 10 & 45 \\
$\mathrm{pN}+$ & 7 & 18 & 25 \\
Total no. of pts & 42 & 28 & 70 \\
\hline $\mathbf{N}=$ lymph node. & & & \\
\hline
\end{tabular}


Table IV. Unadjusted analysis using the Cox's model for overall survival (OS) and recurrence-free survival (RFS) of prognostic factors in the intent-to-treat population $(n=74)^{a}$

\begin{tabular}{|c|c|c|c|c|c|c|}
\hline \multirow[t]{2}{*}{$\overline{\text { Variable }}$} & \multicolumn{3}{|l|}{ OS } & \multicolumn{3}{|l|}{ RFS } \\
\hline & critical events [n/N] & $\mathrm{HR}(95 \% \mathrm{Cl})$ & p-value & critical events [n/N] & $\mathrm{HR}(95 \% \mathrm{Cl})$ & $p$-value \\
\hline \multicolumn{7}{|l|}{ Age group } \\
\hline$<50 y$ & $4 / 38$ & 1 & $p=0.39$ & $9 / 38$ & 1 & $p=0.40$ \\
\hline$\geq 50 y$ & $2 / 36$ & $0.48(0.09,2.7)$ & & $6 / 36$ & $0.64(0.23,1.8)$ & \\
\hline \multicolumn{7}{|c|}{ Baseline nodal status } \\
\hline $\mathrm{N}-$ & $2 / 42$ & 1 & $p=0.15$ & $3 / 42$ & 1 & $p=0.001$ \\
\hline $\mathrm{N}+$ & $4 / 29$ & $3.25(0.59,17.9)$ & & $11 / 29$ & $6.5(1.8,23.3)$ & \\
\hline \multicolumn{7}{|c|}{ Baseline clinical stage } \\
\hline 1 & $0 / 1$ & - & & $0 / 1$ & - & \\
\hline ॥ & $2 / 58$ & 1 & $p=0.004$ & $8 / 58$ & 1 & $p=0.005$ \\
\hline III & $4 / 12$ & $3.2(1.4,7.6)$ & & $6 / 12$ & $4.9(1.7,14.3)$ & \\
\hline \multicolumn{7}{|c|}{ Baseline histologic Elston and Ellis grade ${ }^{[24]}$} \\
\hline 1 & $1 / 8$ & 1 & $p=0.33$ & $1 / 8$ & 1 & $p=0.38$ \\
\hline 2 & $2 / 33$ & $0.32(0.03,3.8)$ & & $5 / 33$ & $0.92(0.11,8.1)$ & \\
\hline 3 & $3 / 17$ & $1.1(0.11,11.3)$ & & $5 / 17$ & $2.11(0.24,18.5)$ & \\
\hline \multicolumn{7}{|c|}{ Estrogen receptor status } \\
\hline Negative & $3 / 31$ & 1 & $p=0.75$ & $8 / 31$ & 1 & $p=0.35$ \\
\hline Positive & $3 / 43$ & $0.77(0.15,3.9)$ & & $7 / 43$ & $0.62(0.22,1.7)$ & \\
\hline \multicolumn{7}{|c|}{ Progesterone receptor status } \\
\hline Negative & $5 / 46$ & 1 & $p=0.23$ & $11 / 46$ & 1 & $p=0.27$ \\
\hline Positive & $1 / 28$ & $0.27(0.03,2.4)$ & & $4 / 28$ & $0.53(0.17,1.7)$ & \\
\hline \multicolumn{7}{|l|}{ Pathologic response } \\
\hline Complete response & $0 / 16$ & 1 & $p=0.16$ & $2 / 16$ & 1 & $p=0.32$ \\
\hline Residual tumor & $6 / 57$ & $\infty(0, \infty)$ & & $12 / 57$ & $2.1(0.47,9.5)$ & \\
\hline \multicolumn{7}{|c|}{ Baseline triple-negative status } \\
\hline non-TNBC & $3 / 51$ & 1 & $p=0.30$ & $8 / 51$ & 1 & $p=0.26$ \\
\hline TNBC & $3 / 22$ & $2.3(0.46,11.4)$ & & $6 / 22$ & $1.8(0.63,5.2)$ & \\
\hline
\end{tabular}

a Bold values are statistically significant.

$\mathbf{H R}=$ hazard ratio; $\mathbf{N}$ = lymph node; $\mathbf{T N B C}=$ triple-negative breast cancer; - indicates estimation not feasible because of low sample size in the considered categories.

(table II). The 3- and 5-year estimates of OS were $95 \%$ and $81 \%$, respectively, and of RFS were $84 \%$ and $65 \%$, respectively. Notably, there were no statistically significant differences between patients with TNBC or with non-TNBC in terms of survival parameters (table II).

We conducted a univariate analysis to identify prognostic factors for OS and RFS (table IV). Baseline lymph node involvement or advanced disease were prognostic of poorer RFS, and baseline high clinical stage was prognostic of poorer OS. However, pCR did not appear to be a prognostic factor for either RFS or OS.
In patients with TNBC achieving a pCR, OS was similar to that of patients without TNBC with a pCR (5-year estimates of $\mathrm{OS}=100 \%$ for both). Among patients without a pCR, there was a trend towards lower OS in patients with TNBC than non-TNBC (3- and 5-year OS estimates were $84 \%$ and $42 \%$ for TNBC, and $97 \%$ and $82 \%$ for non-TNBC; $p=0.07$ ) but the difference was not significant. Similarly, among patients with a pCR, the RFS of patients with TNBC was no different from that in patients with non-TNBC (3- and 5-year RFS estimates were 100\% and 75\% for TNBC and, $100 \%$ and $67 \%$ for non-TNBC; 
HR 0.6; 95\% CI 0.03, 8.8; Log rank test: $\mathrm{p}=0.66$ ). Notably, however, of those patients without a pCR, RFS rates were significantly lower in patients with TNBC than those with non-TNBC (3- and 5-year RFS estimates were $62 \%$ and $62 \%$ for TNBC, and $89 \%$ and $79 \%$ for non-TNBC; HR 3.3; 95\% CI 0.99, 10.8; Log rank test: $p=0.04$ ) [figure 2].

\section{Safety}

Chemotherapy was discontinued before all six cycles were administered because of toxicity of at least grade 3 severity in four patients and of grade 1 or 2 severity in a further four patients, but all eight received surgery as per the study protocol (after a median of four [range of two to five] cycles of treatment).

Treatment-emergent adverse events are summarized in table $\mathrm{V}$, of which neutropenia was the most frequently reported. Grade 4 febrile neutropenia occurred in two patients. Two patients experienced grade 2 peripheral neuropathy related to docetaxel treatment. Interstitial pneumopathy of grade 3 severity occurred in one patient and was considered to be related to docetaxel (table V), but did not lead to respiratory failure. Only one patient developed a grade 3 cardiotoxicity (acute heart failure), which was considered to be related to epirubicin.

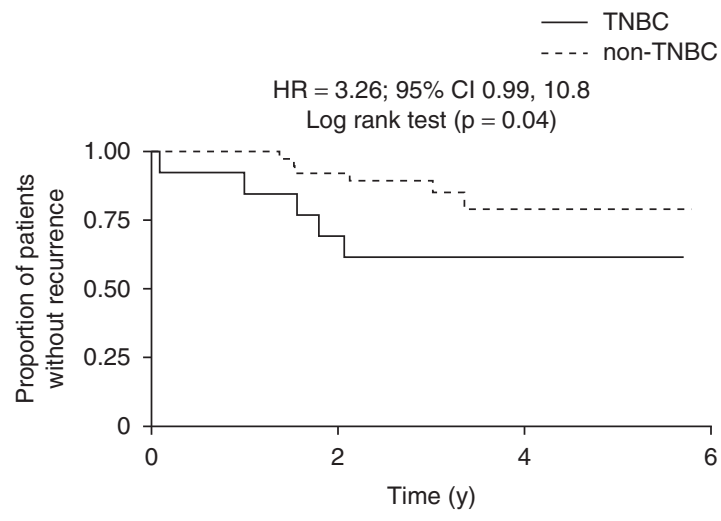

Fig. 2. Recurrence-free survival in patients with breast cancer without a pathologic complete response after neoadjuvant treatment with gemcitabine plus docetaxel alternating with vinorelbine plus epirubicin according to triple-negative status. $\mathbf{H R}=$ hazard ratio; TNBC = triple-negative breast cancer.
Table V. No. of treatment-emergent adverse events in the safety population $(\mathrm{n}=74)$

\begin{tabular}{|c|c|c|c|c|}
\hline \multirow[t]{2}{*}{ Adverse event } & \multicolumn{4}{|c|}{$\mathrm{NCl}$ CTC Grade } \\
\hline & $\overline{1}$ & 2 & 3 & 4 \\
\hline \multicolumn{5}{|c|}{ Hematologic toxicities } \\
\hline Anemia & 7 & 6 & 0 & 0 \\
\hline Neutropenia & 15 & 10 & 28 & 12 \\
\hline $\begin{array}{l}\text { Febrile } \\
\text { neutropenia }\end{array}$ & 0 & 0 & 0 & 2 \\
\hline Fever & 4 & 1 & 0 & 0 \\
\hline Thrombopenia & 7 & 5 & 0 & 0 \\
\hline \multicolumn{5}{|c|}{ Non-hematologic toxicities } \\
\hline Diarrhea & 3 & 0 & 0 & 1 \\
\hline Skin eruption & 10 & 6 & 0 & 0 \\
\hline Nausea/vomiting & 13 & 6 & 0 & 1 \\
\hline Mucositis & 11 & 4 & 0 & 0 \\
\hline Alopecia & 3 & 6 & & \\
\hline Allergy & 5 & 1 & 2 & 0 \\
\hline Neuropathy & 3 & 2 & 0 & 0 \\
\hline Asthenia & 6 & 7 & 0 & 0 \\
\hline Other & 17 & 8 & $3^{a}$ & 0 \\
\hline \multicolumn{5}{|c|}{$\begin{array}{l}\text { a One patient had a cardiotoxicity (acute heart failure), one patient } \\
\text { had phlebitis, one had idiopathic interstitial pneumopathy. }\end{array}$} \\
\hline \multicolumn{5}{|c|}{$\mathbf{N C l} \mathbf{C T C}=$ National Cancer Institute Common Toxicity Criteria, v.2. ${ }^{[22]}$} \\
\hline
\end{tabular}

Treatment was delayed by 1 week for 46 patients $(62 \%)$.

In addition, about one-fifth of patients had more than one adverse event of at least grade 3 severity $(n=14)$ and one-half of patients experienced at least one such adverse event $(n=39)$. Four patients experienced serious treatmentrelated adverse events; two cases of febrile neutropenia, one case of allergic reaction, and one case of pneumonia (the latter related to docetaxel treatment). There were no treatment-related deaths.

\section{Discussion}

Our patients had operable, non-inflammatory, clinical stage II-III breast cancer, of whom most had HER2-negative (HER2-) disease. After treatment with two to three cycles each of alternating gemcitabine plus docetaxel followed by vinorelbine plus epirubicin, about one-fifth of patients had a complete response and survival rates were high (median follow-up 3.18 years). 
The pCR rate falls within the range reported in other neoadjuvant chemotherapy trials in patients with operable breast cancer $(6-29 \%),{ }^{[4]}$ is similar to that reported after the commonly used neoadjuvant combination of docetaxel plus doxorubicin plus cyclophosphamide in a similar patient population $(21 \%),{ }^{[17]}$ and is higher than that reported for concomitantly administered neoadjuvant combination regimens using a 3- or 4-weekly dosing schedule $(12-15 \%){ }^{[3,25,26]}$ However, caution should be applied when making such comparisons because of differences in patient populations and in definitions of pCR across studies.

Recent studies assessing sequential and/or alternating chemotherapy have generally been in the adjuvant setting. ${ }^{[16,27]}$ In one study, investigators concluded that sequential chemotherapy allows dose intensity to be maintained, ${ }^{[16]}$ and our study results support this. Our use of a dosedense regimen is, in turn, supported by their results with a combination of doxorubicin, cyclophosphamide, and paclitaxel given in a 2-weekly regimen that did not adversely affect the risk of local recurrence of disease and significantly $(p<0.05)$ improved OS and disease-free survival compared with a 3-weekly regimen. ${ }^{[16]}$ No such therapeutic advantage was shown in a neoadjuvant setting comparing dose-dense epirubicin plus cyclophosphamide with standard fluorouracil plus epirubicin plus cyclophosphamide in patients with locally advanced or inflammatory breast cancer. ${ }^{[28]}$ However, our dose-dense regimen was associated with good OS and RFS, and local disease recurrence occurred only in $1.4 \%$ of patients.

In our exploratory analysis, baseline clinical stage was prognostic of OS and RFS, baseline tumor grade of OS, and baseline lymph node status of RFS. We determined nodal response, which was observed in $36 \%$ of our patients. This good lymph node response may have contributed, in part, to the good survival outcomes in our study, because negative axillary nodes after neoadjuvant therapy predict favorable long-term outcomes. ${ }^{[23]}$

Approximately one-third of our patients had TNBC, which is higher than expected since TNBCs typically comprise $15 \%$ of breast cancer cases. ${ }^{[15]}$ Our enrollment was slightly skewed in favor of TNBC because we aimed to exclude patients with HER2+ disease, yet we did not select based on hormone receptor status in addition to this (i.e. we did not preselect for TNBC). We postulate that due to the aggressive nature of TNBC, and that these tumors are often larger, ${ }^{[6-8]}$ patients with TNBC are more likely to be candidates for neoadjuvant therapy. To our knowledge, there is no direct evidence in the literature to support this, but two studies in patients with stage II-III breast cancer receiving neoadjuvant therapy reinforce our hypothesis because they too reported about one-third of patients with TNBC. ${ }^{[10,13]}$

It has been suggested that patients with TNBC are candidates for more aggressive chemotherapy. ${ }^{[29]}$ Our dose-dense alternating sequence of anthracycline- and taxane-based combinations showed significantly better activity in patients with TNBC than in those with non-TNBC (pCR $40.9 \%$ vs $14.0 \%$; $\mathrm{p}=0.028$ ). Our results are concordant with several studies demonstrating a better response to neoadjuvant therapy in TNBC than non-TNBC. ${ }^{[10,11,13,14]}$ The anti-tumor activity of our regimen in patients with TNBC was also associated with improved long-term prognosis approaching that of patients without TNBC; between-group differences in OS or RFS were not significant. In contrast to our results, other studies have found that despite a higher pCR in patients with TNBC, the risk of death and relapse was significantly higher in TNBC than patients without TNBC, ${ }^{[10,11]}$ or in TNBC (basallike tumor subtype) than in luminal tumor subtypes. ${ }^{[13]}$ Progression-free survival was $63 \%$ in TNBC versus $76 \%$ in non-TNBC (HR 1.86; $95 \%$ CI $1.39,2.50)$ after neoadjuvant therapy in one such study, ${ }^{[1]}$ but importantly, this difference was no longer apparent after more than 3 years of follow-up (corresponding to our median followup time). Our exploratory analyses revealed that improved survival in patients with TNBC was exclusively in those who had a pCR. Amongst patients without a $\mathrm{pCR}$, the prognosis of patients with TNBC was significantly $(\mathrm{p}=0.04)$ poorer than patients without TNBC (figure 2). These observations are concordant with previous reports, ${ }^{[11,13]}$ for 
example, Carey et al. ${ }^{[13]}$ found that patients with basal-like tumors with residual disease have higher relapse rates and poorer prognosis than those without basal-like tumors who did not achieved a pCR after anthracycline-based neoadjuvant therapy.

Numerous study limitations must be considered when interpreting our results, including the small patient population, relatively short followup, lack of molecular/hormone receptor data for all patients, and that the study was powered to examine $\mathrm{pCR}$, making all other analyses exploratory in nature. Our inclusion criteria were not strictly adhered to, as nine patients were HER2+. Despite these limitations, we were able to demonstrate that a dose-dense taxane-based regimen, with therapy delivered over a shorter total period of time, allows for sequential administration of a dose-dense anthracycline-based regimen, which may help to limit resistance. ${ }^{[15]}$

Only one patient experienced a severe treatment-related cardiotoxic adverse event. During follow-up, there was no late cardiotoxicity, secondary acute myelogenous leukemia, or myelodysplastic syndrome; however, longer follow-up may be required before such adverse events become apparent. No patients experienced severe neuropathy and there were only two serious or severe treatment-related pulmonary adverse events (grade 3 interstitial pneumopathy and serious pneumonia). As expected, neutropenia was the most frequently reported toxicity overall, and the most common severe adverse event despite filgrastim support. However, no patients were hospitalized for neutropenia or febrile neutropenia. We conclude that overall, the majority of adverse events were manageable and, given that there were no treatment-related deaths, this four-drug regimen was associated with an acceptable tolerability profile.

\section{Conclusion}

A regimen of dose-dense combinations of gemcitabine plus docetaxel and vinorelbine plus epirubicin, given in an alternating sequence was associated with favorable survival rates in patients with TNBC, but only in those patients with a pCR Dose intensity was conserved with just three cycles of each combination to produce a good $\mathrm{pCR}$ rate and an acceptable tolerability profile. We recommend that this regimen be further explored in a prospective study in patients with TNBC.

\section{Acknowledgments}

We thank Tracy Harrison and Jenna Mitchell of inScience Communications, a Wolters Kluwer business, who provided medical writing and journal styling assistance, respectively, which was funded by Association pour la Recherche sur les Therapeutiques Innovantes en Cancerologie, Paris, France.

The study was sponsored by Association pour la Recherche sur les Therapeutiques Innovantes en Cancerologie, Paris, France. The study was independently designed, conducted, and managed. Data were also independently collected and analyzed. Preparation, review, and approval of the manuscript were done by all authors, independently of the sponsor.

All authors certify that no actual or potential conflict of interest in relation to this article exists, notably any financial or personal relationships with other people or organizations that could inappropriately influence (bias) their work.

\section{References}

1. Bafaloukos D. Neo-adjuvant therapy in breast cancer. Ann Oncol 2005; 16 Suppl. 2: ii174-81

2. Hennessy B, Hanrahan E, Valero V. Neoadjuvant therapy of breast cancer. Am J Cancer 2006; 5: 411-25

3. Van Praagh I, Cure H, Leduc B, et al. Efficacy of a primary chemotherapy regimen combining vinorelbine, epirubicin, and methotrexate (VEM) as neoadjuvant treatment in 89 patients with operable breast cancer. Oncologist 2002; 7 : 418-23

4. Mieog J, van der Hage J, van de Velde C. Preoperative chemotherapy for women with operable breast cancer. Cochrane Database Syst Rev 2007 Apr 18; (2): CD005002

5. Dent R, Trudeau M, Pritchard KI, et al. Triple-negative breast cancer: clinical features and patterns of recurrence. Clin Cancer Res 2007; 13: 4429-34

6. Liu Z, Liu G, Yang W, et al. Triple-negative breast cancer types exhibit a distinct poor clinical characteristic in lymph node-negative Chinese patients. Oncol Rep 2008; 20: 987-94

7. Rakha EA, El-Sayed ME, Green AR, et al. Prognostic markers in triple-negative breast cancer. Cancer 2007; 109: 25-32

8. Tian XS, Cong MH, Zhou WH, et al. Clinicopathologic and prognostic characteristics of triple-negative breast cancer. Onkologie 2008; 31: 610-4

9. Carey LA, Perou CM, Livasy CA, et al. Race, breast cancer subtypes, and survival in the Carolina Breast Cancer Study. JAMA 2006; 295: 2492-502

10. Keam B, Im SA, Kim HJ, et al. Prognostic impact of clinicopathologic parameters in stage II/III breast cancer treated with neoadjuvant docetaxel and doxorubicin chemotherapy: paradoxical features of the triple negative breast cancer. BMC Cancer 2007; 7: 203 
11. Liedtke C, Mazouni C, Hess K, et al. Response to neoadjuvant therapy and long-term survival in patients with triplenegative breast cancer. J Clin Oncol 2008; 26: 1275-81

12. Rodriguez-Pinilla SM, Sarrio D, Honrado E, et al. Prognostic significance of basal-like phenotype and fascin expression in node-negative invasive breast carcinomas. Clin Cancer Res 2006; 12: 1533-9

13. Carey LA, Dees EC, Sawyer L, et al. The triple negative paradox: primary tumor chemosensitivity of breast cancer subtypes. Clin Cancer Res 2007; 13: 2329-34

14. Rouzier R, Perou CM, Symmans WF, et al. Breast cancer molecular subtypes respond differently to preoperative chemotherapy. Clin Cancer Res 2005; 11: 5678-85

15. Cleator S, Heller W, Coombes RC. Triple-negative breast cancer: therapeutic options. Lancet Oncol 2007; 8: 235-44

16. Citron ML, Berry DA, Cirrincione C, et al. Randomized trial of dose-dense versus conventionally scheduled and sequential versus concurrent combination chemotherapy as postoperative adjuvant treatment of node-positive primary breast cancer: first report of Intergroup Trial C9741/Cancer and Leukemia Group B Trial 9741. J Clin Oncol 2003; 21: 1431-9

17. von Minckwitz G, Kummel S, Vogel P, et al. Intensified neoadjuvant chemotherapy in early-responding breast cancer: phase III randomized GeparTrio study. J Natl Cancer Inst 2008; 100: 552-62

18. Schneeweiss A, Huober J, Sinn HP, et al. Gemcitabine, epirubicin and docetaxel as primary systemic therapy in patients with early breast cancer: results of a multicentre phase I/II study. Eur J Cancer 2004; 40: 2432-8

19. Simon R. Optimal two-stage designs for phase II clinical trials. Control Clin Trials 1989; 10: 1-10

20. European Organisation for Research and Treatment of Cancer. RECIST version 1.0. 2000 [online]. Available from URL: http://www.eortc.be/Recist/Default.htm [Accessed 2009 Dec 17]

21. Hammond MEH, Hayes DF, Dowsett M, et al. American Society of Clinical Oncology/College of American Pathologists guideline recommendations for immunohistochemical testing of estrogen and progesterone receptors in breast cancer. J Clin Oncol 2009; 28: 2784-95

22. National Cancer Institute. Common Toxicity Criteria Manual 1 June 1999 version 2.0 [online]. Available from
URL: http://ctep.cancer.gov/protocoldevelopment/electro nic_applications/docs/ctcv20_4-30-992.pdf [Accessed 2008 Dec 17]

23. Rastogi P, Anderson SJ, Bear HD, et al. Preoperative chemotherapy: updates of National Surgical Adjuvant Breast and Bowel Project Protocols B-18 and B-27. J Clin Oncol 2008; 26: 778-85

24. Robbins P, Pinder S, de Klerk N, et al. Histological grading of breast carcinomas: a study of interobserver agreement. Hum Pathol 1995; 26: 873-9

25. Chua S, Smith IE, A'Hern RP, et al. Neoadjuvant vinorelbine/ epirubicin (VE) versus standard adriamycin/cyclophosphamide (AC) in operable breast cancer: analysis of response and tolerability in a randomised phase III trial (TOPIC 2). Ann Oncol 2005; 16: 1435-41

26. Conte PF, Donati S, Gennari A, et al. Primary chemotherapy with gemcitabine, epirubicin and taxol (GET) in operable breast cancer: a phase II study. Br J Cancer 2005; 93: 406-11

27. Fountzilas G, Skarlos D, Dafni U, et al. Postoperative dosedense sequential chemotherapy with epirubicin, followed by $\mathrm{CMF}$ with or without paclitaxel, in patients with highrisk operable breast cancer: a randomized phase III study conducted by the Hellenic Cooperative Oncology Group. Ann Oncol 2005; 16: 1762-71

28. Therasse P, Mauriac L, Welnicka-Jaskiewicz M, et al. Final results of a randomized phase III trial comparing cyclophosphamide, epirubicin, and fluorouracil with a doseintensified epirubicin and cyclophosphamide + filgrastim as neoadjuvant treatment in locally advanced breast cancer: an EORTC-NCIC-SAKK multicenter study. J Clin Oncol 2003; $21: 843-50$

29. Mehta RS. Dose-dense and/or metronomic schedules of specific chemotherapies consolidate the chemosensitivity of triple-negative breast cancer: a step toward reversing triple-negative paradox [letter]. J Clin Oncol 2008; 26: 3286-8; author reply 3288

Correspondence: Dr Jacques Medioni, Département d'Oncologie Médicale, Hôpital Européen Georges Pompidou, 20, rue Leblanc, Cedex 15, 75908, Paris. E-mail: jacques.medioni@egp.aphp.fr 